\title{
Strangulated stomach within a femoral hernia
}

\author{
RICHARD J. CADE* \\ F.R.A.C.S., F.R.C.S.
}

\author{
ROBERT H. S. LANE \\ M.S., F.R.C.S.
}

Royal Hampshire County Hospital, Winchester

\begin{abstract}
Summary
A complication of femoral hernia not previously described, namely gastric strangulation, is reported.
\end{abstract}

KEY WORDS: haematemesis, pharyngeal pouch, chronic bronchitis.

\section{Case report}

A 79-year-old lady was admitted to hospital following a haematemesis. At that time she was also noted to have an irreducible, non-tender femoral hernia. Endoscopy was attempted, but abandoned as it was thought she might have a pharyngeal pouch. Barium swallow and meal confirmed the presence of a pouch but was otherwise normal.

The patient was discharged only to be readmitted 3 months later with an enlarging femoral hernia, abdominal pain and vomiting. She was hypotensive, peripherally vasoconstricted and extremely dyspnoeic with evidence of chronic obstructive airways disease. There was a massive left femoral hernia which was irreducible and tender. There were no significant abdominal signs. A naso-gastric tube was passed and 2 litres of 'coffee-ground' fluid aspirated. Concomitantly there was a marked reduction in the size of the hernia.

At laparotomy the stomach was seen to be incarcerated in the hernia. When the hernia was reduced, the anterior wall of the stomach was found to be necrotic over an area of approximately 8 by 5 $\mathrm{cm}$ with a more extensive area of mucosal damage. It was noted that she was visceroptopic and there was scarring of the small intestine, mesentery, omentum, right colon and appendix. The necrotic anterior gastric wall was excised and the defect closed. The neck of the hernial sac admitted four fingers but as the patient was profoundly hypotensive at this stage a simple closure of the femoral ring was all that was deemed advisable, the sac itself being left in situ.

She recovered well postoperatively but she developed a recurrent hernia which subsequently required

*Present address: Department of Thoracic Surgery, Royal Devon and Exeter Hospital (Wonford), Exeter EX2 5DS. a definitive repair through a combined high and lowi approach.

\section{Discussion}

The herniation of omentum, small intestine, color appendix (Duari, 1966; Voitk, Macfarlane and E\& trada, 1974; Jacobson, 1978) and fallopian tube and ovary (King, 1978) in femoral hernias have all beem well described. The stomach may occasionally be included in the contents of an inguinal hernia and. very rarely becomes strangulated, three cases having been reported (Gue, 1970; Padmanabhan and Low enfels, 1976; Nagendran, 1977). This complicatiotb has not been recorded in a femoral hernia. Thiso patient's visceroptopic peculiarities and the very de femoral ring presumably permitted the abdominat viscera, including the stomach, to migrate into the hernial sac. In retrospect, the previous admission t hospital is also of interest as it is likely that the haematemesis was due to another episode of gastrie strangulation in the hernia but which spontaneousl $\vec{\delta}$ reduced.

\section{References}

DUARI, M. (1966) Strangulated femoral hernia-a Richter's type containing caecum and base of appendix. Postgraduate Medic Journal, 42, 726.

GUE, S. (1970) Spontaneous rupture of stomach. A rare complicatiof of inguinal hernia. British Journal of Surgery, 57, 154.

JACOBSON, S. (1978) Mucocele of the appendix: an unusual finding at exploration of right-sided inguinal pain. Acta Chirurgieg Scandinavica, 144, 557.

KING, R. (1978) Congenital, strangulated femoral hernia in an infa aged six months containing the ovary and fallopian tube. British Journal of Clinical Practice, 32, 209.

NAGENDRAN, T. (1977) Stomach contained in a giant scrotal hernia. American Journal of Surgery, 43, 473.

Padmanabhan, A.I. \& Lowenfels, A.B. (1976) Letter: An unusual hernia. Journal of the American Medical Association, 235, 2813. W

VoITK, A.J., MACFARLANE, J.K. \& EstraDA, R.L. (1974) Rupture appendicitis in femoral hernias: report of 2 cases and review of the literature. Annals of Surgery, 179, 24.

(Accepted 1 June 1983) 